\title{
Deposition and Dissolving of Silver Nanowires for micro circuits
}

\author{
Wang Jing ${ }^{1,2,3, a}$, Wu Zhe Z b $^{3, \text { b }}$ \\ ${ }^{1}$ Departmen of Electronic Science and Engineering, Nanjing university of Posts and \\ Telecommunications, Nanjing, 210003, China \\ 2 Jiangsu Provincial Engineering Laboratory for RF Integration and Micropackaging, Nanjing, \\ 210003, China \\ ${ }^{3}$ Department of Physics, Nanjing university, Nanjing, 210093, China \\ aemail: IreneWang@njupt.edu.com, bemail:Wuzh1223@163.com
}

Keywords: micro circuitry; polarized field; electrochemical dissolve; silver nanowire; ultra thin liquid layer

\begin{abstract}
According to the principle of electric field polarization, we sputtered one or a few silver squares on substrate to strengthen polarization of local electric field in the depositing system of silver nanowires in an ultra thin liquid layer. In this paper, we observed electrochemical dissolving inside sputtered silver plates and in deposited silver wires. By monitoring different patterns of silver plates and nanowires at the end of the depositing process, we confirm the possibility of manufacturing special pattern of silver wire only with necessary structure by adjusting an appropriate electric signal.
\end{abstract}

\section{Introduction}

Considering fast developing technology of micro electric circuit manufacture, it requires that more accuracy and stability of cells. Silver is a proper material with its fine electric and heat conductivity. The difficulties including manufacturing micro silver structure accurately, without unnecessary part, have not solved very well, especially in manufacture of silver wires, which is regularly used in micro electric circuits.

In this paper, we construct an electrochemical deposition system to grow silver line. Based on the polarization theory of electric field [1] [2] [3] , we modify the substrate with silver plates adhered to change the polarization of electric field, which help understanding the mechanics of electrochemical dissolving in metal deposition. We control electric signals adding on an ultrathin liquid film system [4] to deposit silver nano wires. By adjusting added electric signals in different silver pattern systems [5] [6], we obtain several morphologies of silver micro structure, which suggests the possibility to eliminate unnecessary micro structure in the process of manufacturing micro circuits.

\section{Depositing and Dissolving Mechanics of silver wires}

The mechanics of electrochemical depositing and dissolving is very simple. Based on electrochemical growing of metal [1] [3], sputtered silver squares are polarized by local electric field. The electric charges assemble near the square end. By the polarizing orientating, cathode or anode reaction will happen as follows, respectively:

a) near the platinum line anode side, silver ions get electrons and deposit to silver wires:

$\mathrm{Ag}^{+}+e^{-} \rightarrow \mathrm{Ag}$

b) near the platinum line cathode side, silver atoms of the central silver square lose electrons, transforming to silver ions into the electrolyte:

$\mathrm{Ag}-\mathrm{e}^{-} \rightarrow \mathrm{Ag}^{+}$

On the basis of the principle above, silver in the ultra thin electrolyte film deposits or dissolves according to the polarizing condition of local electric field. 


\section{Experiment Design}

We prepare a piece of clean cover glass as substrate to deposit silver, with a side length of $18 \mathrm{~mm}$. As illustrated in figure 1 (a), (b) and (c), one or a few silver squares are sputtered on one face of the cover glass, each of which has a side length $500 \mu \mathrm{m}$ and a thickness of $100 \mathrm{~nm}$. Shown in figure 1 (b), the central distance between the two silver square is $1.2 \mathrm{~mm}$, while the central distance of every couple squares is $1.5 \mathrm{~mm}$, shown in figure 1 (c). Electrolyte is dropped on a piece of bottom glass, between two parallel platinum line as electrodes. Then cover the substrate with the sputtered face adown and air bubble kept out, the electrochemical deposition cell is constructed. The electrolyte is prepared with $0.001 \mathrm{~mol} / \mathrm{L} \mathrm{AgNO}_{3}$ solution.

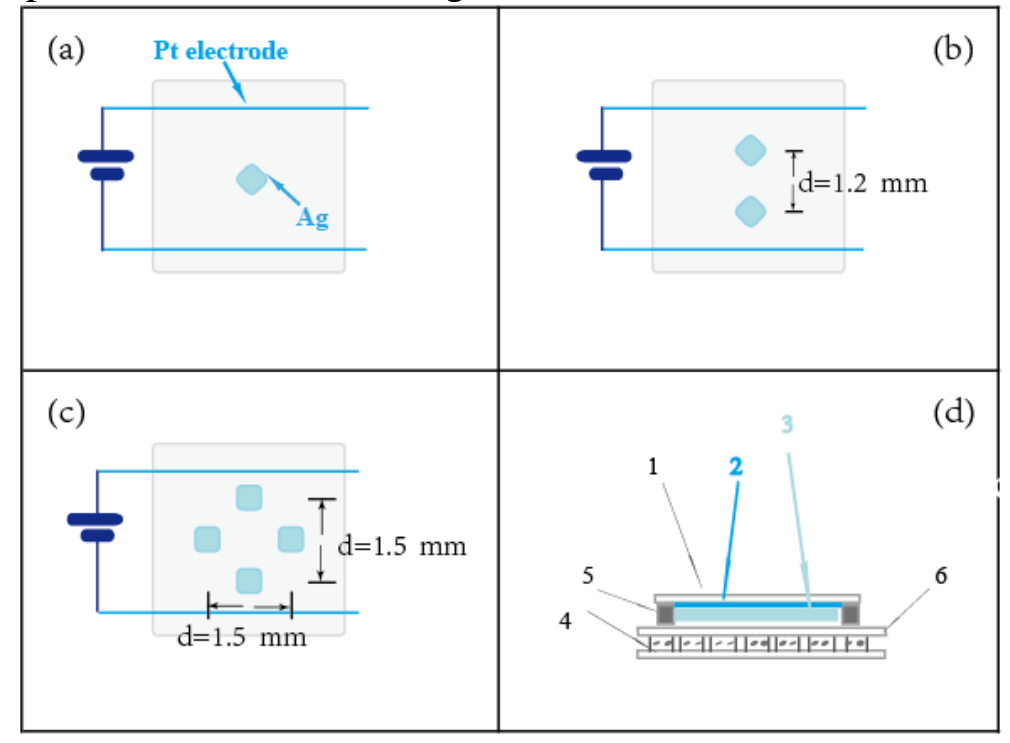

cover glass with (a) 1, (b) 2, (c) 4 silver squares adhered. (d) the electrochemical deposition cell: 1. cover glass 2 . ultra thin liquid film 3 . ice layer 4 . temperature controlling element 5 . platinum electrodes 6 . bottom glass

Fig.1. The illustration of experimental system

The electrochemical deposition cell is laid on a temperature controlling element, as shown in figure 1 (d). With adjusting the current through the element, it is easy to control the temperature of electrochemical deposition cell. By repeatedly increase and decrease the temperature of electrolyte, only one ice nucleus can be obtained, around which water concretes to monocrystal ice, finally only an ultra thin liquid film is left between the ice layer and the cover glass. Then we keep a constant temperature needed by adjusting the current through the element, add electric signals on the electrodes. The silver wires are deposited on the under face of cover glass, which silver squares sputtered on.

Adding different electric signals between two platinum electrodes at different temperature for different reacting time, we can get several results, mainly optic and SEM photos recording the electrochemical process in the ultra thin liquid film. In this paper, we add voltage signals of $2.5 \mathrm{~V}, 4$ $\mathrm{V}$ and $8 \mathrm{~V}$ on silver square system for several tens of minutes, which will be mentioned in the following figures. 


\section{Test results}

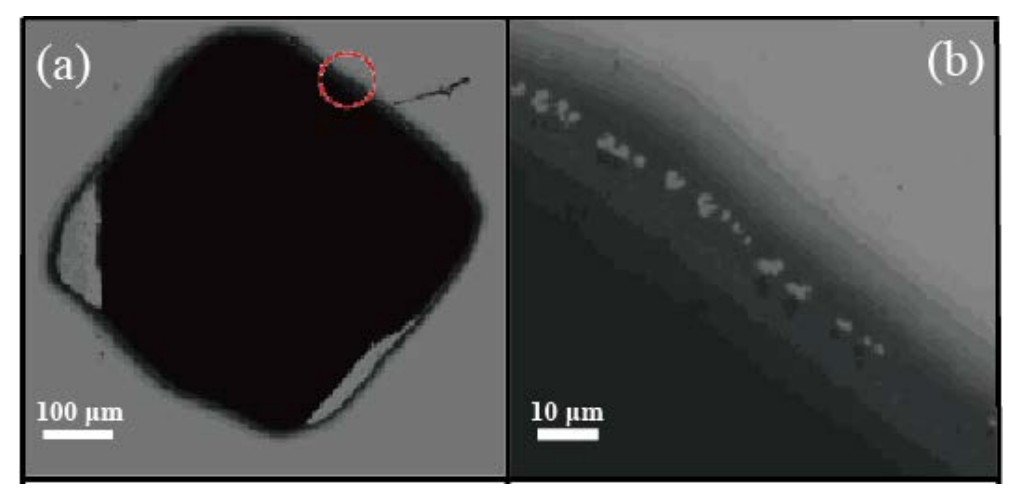

(a)The single silver square depositing to anode and dissolving from cathode $\left(-5.5^{\circ} \mathrm{C}, 8 \mathrm{~V}, 15 \mathrm{~min}\right.$, the top direction is anode) (b) High resolution photo of red circled area in (a), showing the slick sliver sprout spreading to the anode

Fig.2. optic photos of single silver square

After the electrochemical process, the photos of substrates are obtained by optic microscope Nikon Microscope Eclipse 80i. The photograph of single silver square is supplied in figure 2. In figure 2 (a), it is seen that the silver square is not intact any longer. Near the cathode direction the silver square loses some part of it, which turns light at former dark area, responsible to sputtered district. Several times of electrochemical results the same, ruling out mechanical tear, which means that silver atoms of these areas loses electrons and dissolve into the solution. Figure 2 (b) shows an amplified photo of red circled area of figure 2 (a), in which we can see slick silver stretching from the end near anode of the square. In add, the edge of silver square shows two outlines around, which suggests an extra deposition of silver.

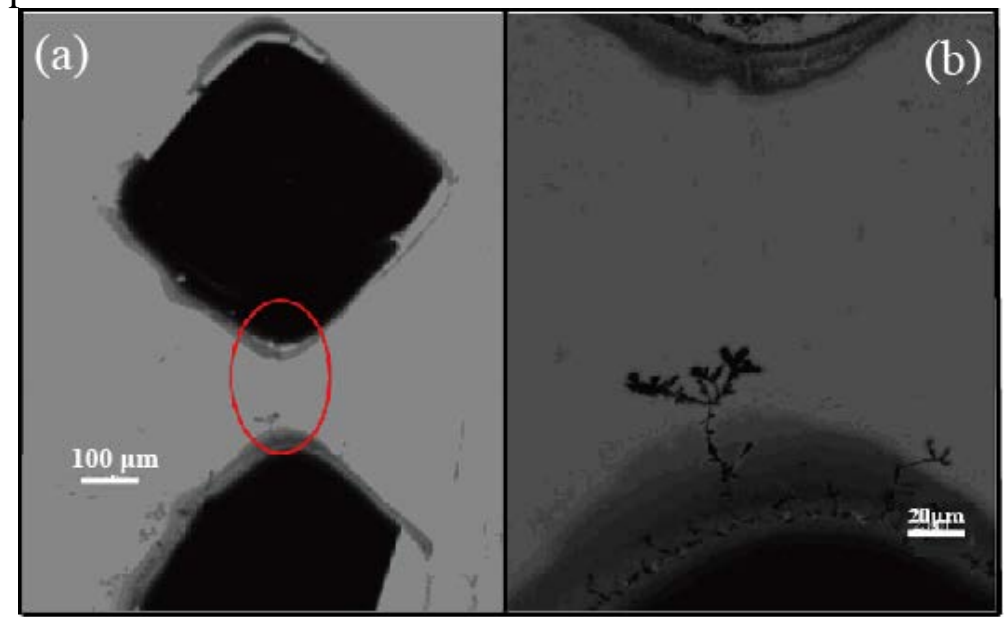

(a)The area between the couple of silver squares, silver depositing and dissolving from cathode to anode direction $\left(-5.5^{\circ} \mathrm{C}, 8 \mathrm{~V}, 15 \mathrm{~min}\right.$, the top direction is anode) circled area in (a)

Fig.3. The optic photos of couple silver squares

In figure 3 it indicates the system of couple silver squares. Figure (a) is an optic photo focused on the area between two squares. The top square has shrunk when electrochemical reaction completes, compared to former area of sputtered silver. The up edge of the bottom square looks vague. Amplified photo in figure 3 (b) indicates that it is a thin layer spreading from the square. In add, there are also several silver branches growing from the end point of square. The bottom edge of top square is not as sharp as before reacting any longer, which indicates that the top silver has shrunk at this end point, meaning dissolving near the cathode. 


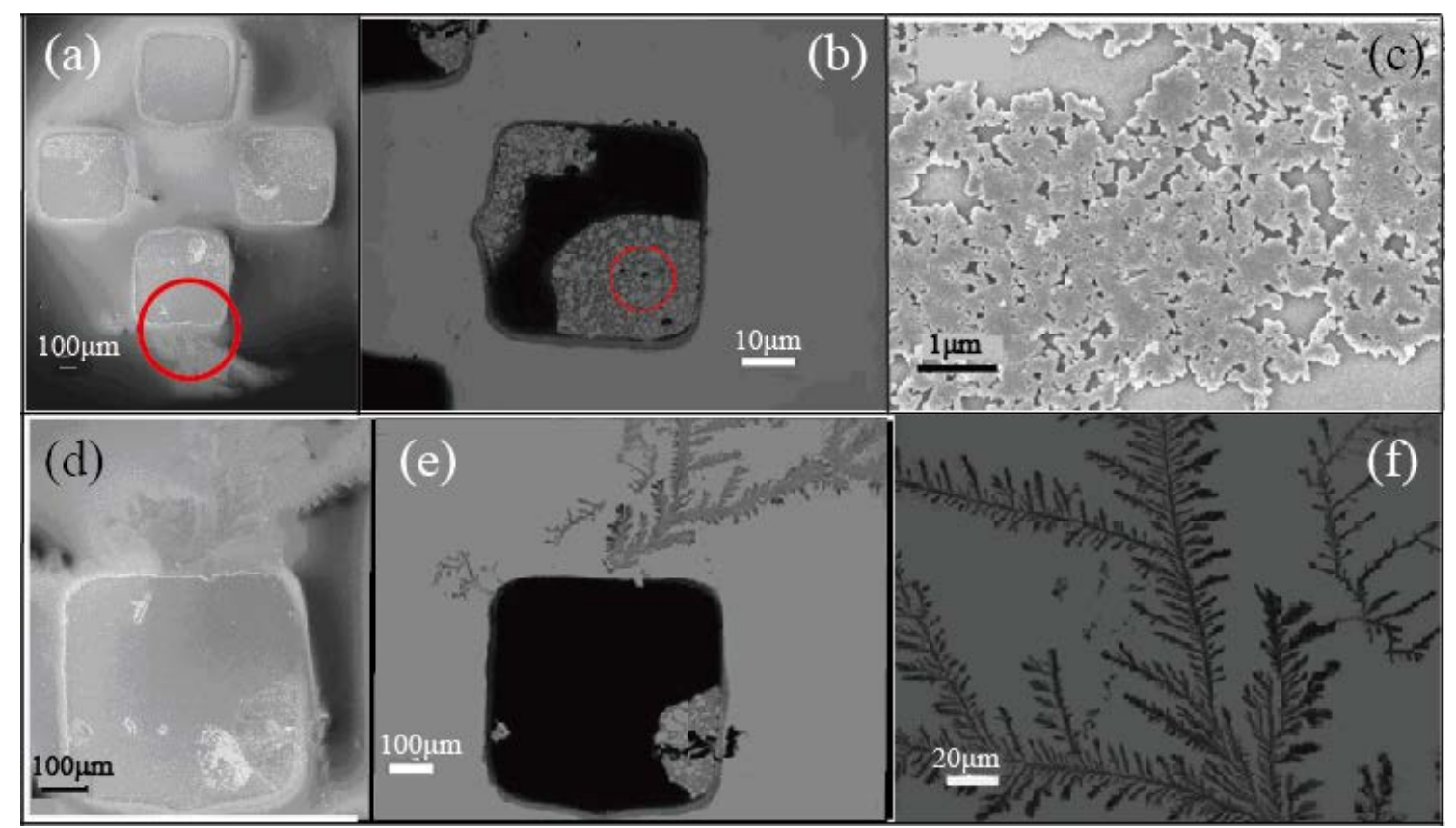

(a)SEM photo of four silver squares $\left(-5.5^{\circ} \mathrm{C}, 8 \mathrm{~V}, 20 \mathrm{~min}\right.$, the top direction is anode) (b) optic photo of silver dissolving in one of the silver squares (the top direction is cathode) (c) High resolution SEM photo of red circled area in (b), (d) Mirrored magnified details of red circled area of (a) (the top direction is cathode), (e) responsible optic photo of (d), (f) the optic details of silver branches spreading from the square (the top direction is cathode) Fig.4 Optic and SEM photos of four silver squares system

The system of four silver square is complicated. Illustrated in the SEM photo of four square sample(taken by scanning electron microscope LEO-1530VP), figure 4 (a), every silver square shrink by varying levels. The top square has kept the main structure of square, but in the other three square there appear severe dissolving that squares cannot maintain their shapes, especially the left and right square. In the parts of the right square both near anode and cathode, there are only several holes left in the area of silver before, as shown in figure 4 (b). The red circled area in figure 4 (b) is magnified in figure 4 (c), which is clearly indicated the hole structure where was silver before electrochemical reacting.

Figure 4 (d) shows the SEM photo of silver wires branching from the end of bottom square near anode, responsible to the red circled area in figure 4 (a). The responsible optic photo is indicated in figure 4 (e). It is clear that there also appears dissolving in the square, which is more obvious compared to dissolving in couple square system shown in figure 2 and 3. The silver wires were deposited to the direction of anode, with several branches which have complete structure, as figure 4 (f) shows.

In the system of four silver squares, we can observe silver wires spreading not only on the silver squares but also on the platinum anode to the cathode direction. This is because the added electric field polarized all the environment, including the part around platinum anode. This phenomenon does not appear in other condition such as couple square system. In add, in four square system, the dissolving of silver square is more serious than other system above. In our opinion, existence of more silver squares result in stronger polarization of local electric field, leading to more obvious deposition and dissolving. 


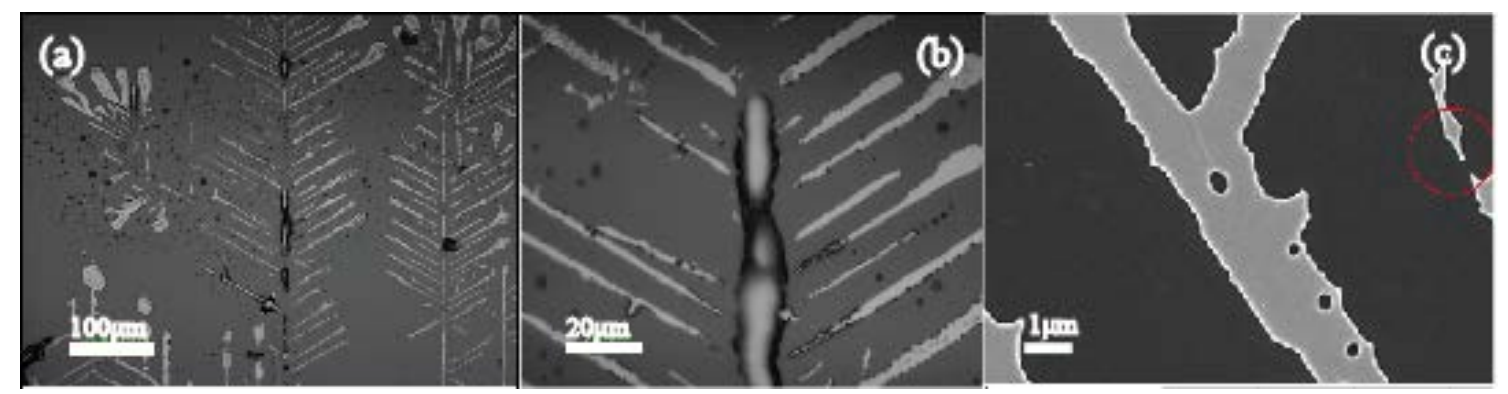

(a) SEM photo of dissolving silver wires (b) amplified SEM photo of one silver wire (c) holes and break inside silver wire

Fig.5. SEM photo of dissolving silver wires $\left(-6^{\circ} \mathrm{C}, 4.5 \mathrm{~V}, 25 \mathrm{~min}\right.$, the top direction is cathode)

We discover dissolving phenomenon at silver wires when the electric signal changed. Under the voltage of $4.5 \mathrm{~V}$ at temperature of $-6^{\circ} \mathrm{C}$, the electrochemical reacting lasts for 25 minutes. There appears dissolving in the silver wires, as figure 5 (a) shows. The SEM photo in figure 5 (b) is amplified from figure 5 (a), where the electrochemical dissolving looks much clearer. Figure 5 (c) shows the delicate structure in a silver wire with high resolution. Distinct holes appear inside the silver branch in the centre of SEM photo, and in the red circled area the silver wire breaks off.

The figure 5 supplies us more confidence that if we adjust an appropriate electric signal, manufacturing special pattern of silver wire only with necessary structure can be succeeded.

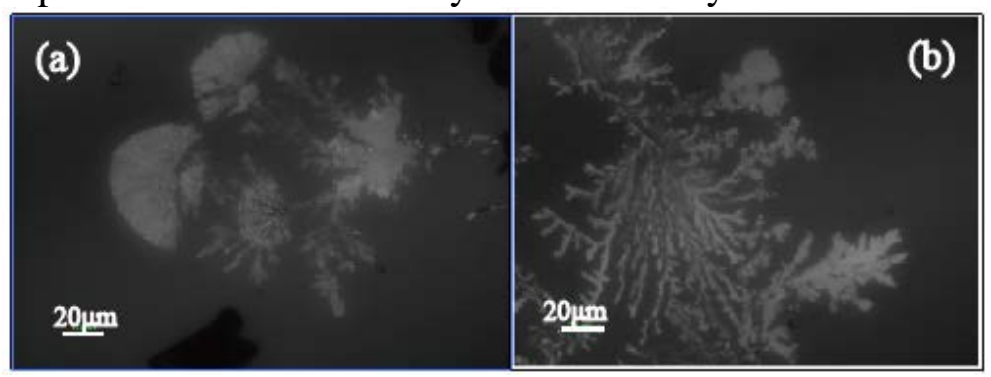

Fig.6. silver deposited at temperature $-5.5^{\circ} \mathrm{C}$ for single silver square pattern under different signal

(a) sine current with an effective value $1 \mathrm{~mA}$, (b) constant voltage of $2.5 \mathrm{~V}$

To compare the influence of different electric signals, we change the added signal between electrodes, while all the other factors are the same as the condition of single silver square. The silver wire spreading from the end of silver square near anode performant different patterns from the one reacted at constant voltage. As shown in figure 6 (a), the silver wires spread and shrink periodically, responding to the sine current signal, which the effective value is $1 \mathrm{~mA}$. We also add a constant voltage signal by $2.5 \mathrm{~V}$, which the silver branches cannot indicate remarkable regulation, as figure 6 (b) shows.

In the experiment we found that there usually appear a lot of silver branches not from squares but from the platinum anode to cathode. Sometimes these branches grow too fast that the deposition and dissolving are not clear enough. To decrease the interfere of branching from platinum anode, the next step is trying similar substrates composed of smaller silver squares. The difficulties to solve is that how to sputter more and smaller silver plates accurately, to understand the depositing and dissolving in manufacturing silver wires for micro circuits.

\section{Conclusion}

We observed electrochemical deposition as well as dissolving at different ends of silver squares. According to optic and SEM photographs of silver, we find that dissolving usually appears at the end of silver square near cathode, and silver wires only spread at the anode end. Infer from the above results, we conclude that out electric field leads to the polarization of silver squares, and the depositing and dissolving happen consequently.

From single silver square to four square system, the phenomenon of depositing and dissolving is more and more serious with the increasing number of silver square. We prospect that more silver squares will cut the uniformity of electric field, bringing stronger polarization of local electric field, 
which the attemption will be completed in our next work.

Another following work is to explore how the asymmetric dissolving of left and right squares in the system of four silver squares happens. The planned experiments include to testify the currency, voltage around the silver squares under different temperatures. It may be necessary to try different patterns of silver plate, such as sputtering smaller squares or circle silver dots, or increase the number of silver plates, etc. . All planned methods above will help us explore the localized electric field, which is very useful to understand the process of electrochemical depositing and dissolving. Based on factors above all, more delicate silver wires could be produced with higher accuracy and stability, which is hopeful applied in the manufacture industry of nano circuit.

\section{Acknowledgement}

In this paper, the research was sponsored by the National Nature Science Foundation (Project No. 51302136) and Youth Fund Project of Nanjing University of Posts and Telecommunications (Project No. NY213017).

\section{References}

[1] Li Di, principles of Electrochemistry. Beijing: Beihang University Press, 1999.

[2] Min Naiben, Physics Basis of Crystal Growth. Beijing: Science Press, 1987.

[3] Fu Xiancai, Physical Chemistry. Nanjing: Nanjing University Press, 2001.

[4] M. Wang, S. Zhong, X.-B. Yin, J.-M. Zhu, R.-W. Peng, Y. Wang, K.-Q. Zhang and N.-B. Ming: Phys.Rev.Lett., 200186 (3827)

[5] Jean-Claude Bradley, Hsuing-Ming Chen,Jeffrey Crawford, Jennifer Eckert, Karima Ernazarova,Thomas Kurzeja, Muduo Lin, Michael McGee,Wolfgang Nadler \& Samuel G. Stephens: Nature 1997 389(268)

[6] Jean-Claude Bradley, Zhongming Ma and Samuel G. Stephens: Adv. Mater. 1999 375(5) 